\title{
Glomerular filtration rate (GFR)
}

National Diabetes Information Clearinghouse (NDIC)

\section{Definitions}

Kidney

Defined by National Diabetes Information Clearinghouse (NDIC)

\section{Source}

National Diabetes Information Clearinghouse (U.S.). (2009). The diabetes dictionary. [Bethesda, Md.]: U.S. Dept. of Health and Human Services, National Institutes of Health, National Institute of Diabetes and Digestive and Kidney Diseases, National Diabetes Information Clearinghouse.

The rate at which the kidneys filter wastes and extra fluid from the blood, measured in milliliters per minute. 